\title{
Superficial Dermatophytic Infection Prevention and Its Management: A Review
}

\author{
Sawood Ahmad', Ghufran Ahmad' ${ }^{2}$, Mohd Mohsin ${ }^{3}$, Mokarram Ali ${ }^{1}$, \\ Musab Ahmad ${ }^{4}$ \\ ${ }^{1}$ MD Scholar Department of Ilmul Advia, Faculty of Unani Medicine, A.M.U., Aligarh, U.P India \\ ${ }^{2}$ Professor Department of Ilmul Advia, Faculty of Unani Medicine, A.M.U., Aligarh, U.P India \\ ${ }^{3}$ Associate Professor Department of Amraz-e-Jild wa Zohrawia, Faculty of Unani Medicine, A.M.U., Aligarh, \\ U.P India \\ ${ }^{4}$ Assistant Professor, Department of Moalejat, Hakim Rais Unani Medical College, Sambhal, U.P, India
}

Corresponding Author: Sawood Ahmad

\begin{abstract}
Dermatophytes are a group of parasitic fungi that live at the expense of the keratin in the skin, nails and hair. They are generally confined to the stratum corneum of the epidermis and skin appendages, particularly in the tropics, where heat and humidity provide the ideal conditions for the growth of fungi that cause cutaneous infections. The dermatophytes that usually cause only superficial infections of the skin are grouped into three genera: Trichophyton, Microsporum, and Epidermophyton. They can be classified into three groups according to their normal habitats: anthropophilic species, zoophilic species and geophilic species. Dermatophyte infections are subclassified in Latin names according to the sites of skin involved, e.g. Tinea faciei, Tinea manuum, Tinea corporis, Tinea cruris, Tinea pedis, Tinea capitis, Tinea unguium. Trichophyton rubrum is the most common cause worldwide for superficial dermatophytosis. By their metabolic activity they produce inflammatory responses in the form of erythema, scaling, postulation and micro-abscess formation giving rise to itching and discomfort. The high prevalence rate of superficial dermatophytic infection has shown that $20-25 \%$ of the world's population. The diagnosis was confirmed on the basis of clinical presentation, $\mathrm{KOH}$ examination of skin and would light examination. The article also discusses the main treatments for superficial dermatophytosis, with a practical approach to the most commonly used topical and systemic drugs, referring also to their dosage and duration
\end{abstract}

of use in different types of the dermatophytic infection.

Keywords: Dermatophytosis; Trichophyton rubrum; Tinea; $\mathrm{KOH}$ examination.

\section{INTRODUCTION}

Superficial fungal infections of the skin are among the most common diseases seen in our daily practice. Dermatophytes are a group of parasitic fungi that live at the expense of the keratin in the skin, nails and hair. They are generally confined to the stratum corneum of the epidermis and skin appendages, particularly in the tropics, where heat and humidity provide the ideal conditions for the growth of fungi that cause cutaneous infections, especially in the moist areas of the body, such as the regions between the toes, groin and below the breasts. ${ }^{[1,2,3,4]}$ Direct contact is sufficient to transmit the infection from a contaminated surface or host to another. However they can be invasive and cause serious widespread infections in immunocompromised patients, with the development of granulomas. [5] With the appearance of AIDS therefore, the incidence of dermatophytosis has increased. Fungi can evade immune system responses via different processes, including recombination, mitosis, and expression of genes involved in oxidative stress responses, and, 
therefore, can cause chronic fungal diseases. Dermatophytosis is commonly designated as 'Tinea' or Ringworm. The main groups of fungi causing superficial fungal infections are dermatophytes, yeasts and moulds. The dermatophytes that usually cause only superficial infections of the skin are grouped into three genera: Trichophyton, Microsporum, and Epidermophyton. They can be classified into three groups according to their normal habitats: humans (anthropophilic species), animals (zoophilic species) and soil (geophilic species). Dermatophytes grow on keratin and therefore cause diseases in body sites wherein keratin is present. These sites include the skin surface, hair and nail. Presence of hyperkeratosis such as palmoplantar keratoderma predisposes to dermatophyte infections. Trichophyton rubrum is the most common cause worldwide for superficial dermatophytosis. By their metabolic activity they produce inflammatory responses in the form of erythema, scaling, postulation and micro-abscess formation giving rise to itching and discomfort. Central clearing is sometimes seen particularly in Tinea corporis. This results in formation of a classic "ringworm" lesion. However, the clinical signs may vary, depending on the part affected. Dermatophytes commonly grow only on the keratinized tissues; but the fungus usually stops spreading when it comes in contact with the living cells or area of an inflammation.

\section{ETIOLOGY}

The main cause of dermatophytosis is dermatophytes, belonging to three generaMicrosporum, Epidermophyton and Trichophyton. They can originate from the soil (Geophilic) or animals (Zoophilic) or be conformed to human skin (Anthropophagic). And several species of these genera impact on humans. Epidermophyton species affect nails and skin, Microsporum species affect hair and skin while Trichophyton species affect hair, nails and skin. Dermatophyte infections are sub classified in Latin names according to the sites of skin involved, e.g. tinea faciei, tinea manuum, tinea corporis, tinea cruris, tinea pedis, tinea capitis and tinea unguium. ${ }^{[6,7,8]}$

\section{ECOLOGY}

The dermatophyte and their congeners have long been divided into the three general ecological groups, based on their reservoir and habitat anthropophilic (humans), geophilic (soil) and zoophilic (animal).

Anthropophilic: These are associated primarily with humans and rarely infect animals. Since transmission is man to man, contracting the disease therefore requires human contact.

Geophilic: These are associated with keratinous materials such as hairs, feathers, hooves, and horns, after these materials have been dissociated from living animals and in the process of decomposition. They may case animal and human infection both. Zoophilic: these are basically animal pathogens, and occasionally infect humans.

\section{EPIDEMIOLOGY}

The high prevalence rate of dermatophytic infection showed that 20$25 \%$ of the world's population has skin mycosis. ${ }^{[9,10]}$ Dermatomycosis is the most common of the mycosis infections worldwide. There has been study rise in the incidence of cutaneous dermatophyte infection and increasing the rate of treatment failure or relapse among the mycotic patients undergoing treatment. ${ }^{[11]}$

\section{GEOGRAPHICAL DISTRIBUTION OF DERMATOPHYTIC INFECTION}

Ringworm or Tinea is the most common and widespread endemic infectious disease (Rippon 1988; Kates et al. 1990; Odom 1993; Guta et al. 2003). In some geographic areas or environments more than $30 \%$ of the population is affected (Rippon 1985; Noguchi et al. 1995) therefore dermatophytosis poses a considerable worldwide health problem. ${ }^{[12]}$ 
Prevalence of various species varies from one century to another century but trichophyton rubrum is the most common dermatophyte infection of the Tinea unguium Tinea corporis, Tinea cruris and Tinea pedis worldwide. ${ }^{[13,14]}$

\section{PATHOGENESIS}

The invasion of the epidermis by the dermatophytes follows a common pattern starting with adherence between arthroconidia and keratinocytes, followed by the penetration through and between cells and the development of host response. Infection may be transmitted by direct contact with infected individuals or animals, by fomites such as clothing, furniture etc. The presence of a suitable environment on the host skin is of critical importance in the development of clinical dermatophytosis.

\section{SKIN}

During the incubation period of 1-3 week the dermatophyte grows in the stratum corneum with minimal clinical stage of infection. The fungus which enters the skin through the minor trauma remains the stratum corneum as it cannot penetrate the deeper layers of skin due to the inhibitory factor in serum. Once the infection is established in stratum corneum, two factors are important in determining the size and the duration of the lesion:

- The rate of the grow of the organism

- The epidermal turnover rate

\section{NAILS}

Onychomycosis commonly begins by invasion of stratum corneum of the hyponychium and distal nail bed, from where the infections moves proximally in the nail bed and invade the ventral surface of the nail plate. ${ }^{[15]}$ Subungual hyperkeratosis result from a hyper proliferative reaction of the nail bed in response the infection.

There are two routes by which dermatophytes could enter into nails:

- Fungal penetrates the eponychium and the hyphae grow toward the nail bed in the comparatively soft keratin of the ventral layer.

- Fungal hyphae enter the ventral nail grooves, follow the lamellar structure of the nail plate transversely and reach the ventral layer. An elaborate network of channels and lacunae may be formed leading to opacity and eventual destruction of the nail plate.

\section{HAIRS}

The fungus invades the hair follicular level from the adjacent stratum corneum. They descend within the intrapilary portion of hair until they reach the border of the keratogenous zone. The terminal tuft of hyphae in this location is termed Adamson's fringe.

Depending on the infecting species, spores are either formed within the hair shaft or on the surface of the hair shaft Tinea tonsurans and Tinea violaceum produce the endothrix infection. Spore formation within the hair in the endothrix infection results in a significant weakening of hair shaft with subsequent fracture of the shaft at the scalp line. The remnant of the hair on the follicle is called 'black dot'. Endothrix infections continue past the anagen phase into the telogen phase producing the more chronic infection. ${ }^{[16]}$

Ectothrix infection of the hair may occur with either small spore producing species with large spore producing species like. The infected hair when plucked breaks at its weakest point which is just above Adamson's fringe. Thus hair grows to its normal length. In keroin, two theories are postulated:

- Results from diffusion of metabolites or toxins from fungus.

- Results from an immunological response to dermatophyte antigen.

\section{CLINICAL PERSENTATION AND CLASSIFICATION}

\section{Anatomical classification}

Glabrous skin (Tinea corporis Tinea cruris, Tinea faciei) 
Skin rich in terminal hair follicles e.g. scalp and beard (Tinea capitis Tinea barbae)

Highly keratinised skin e.g. palms, soles (Tinea pedis and Tinea manuum)

Nail infection (Tinea unguium).

Classification according to site of infection $^{[18,19,20]}$

\section{Site of involvement}

Glabrous skin

Groin

Hair/Scalp

Face

Hands

Feet

Beard area

Nails

\section{Clinical types}

Tinea corporis

Tinea capitis

Tinea faciei

Tinea Manuum

Tinea pedis

Tinea barbae

Tinea unguium
Tinea cruris

Anatomical clinical classification of the dermatophytosis

There are the four broad groups

Dermatophytosis limited to keratinized stricture:

- Tinea corporis, Tinea cruris, Tinea faciei, accompanied or not, by non perforating folliculitis

- Tinea of palm and soles

- Tinea capitis (excluding kerion and favus)

- Onychomycosis

Perforating Dermatophytosis:

- Chronic perforating folliculitis

- Kerion and sycosis

- Favus

- Dermatophytoses actively invading dermal tissue (dermatophytic granuloma)

- Dermatophytic granuloma following invasion of hair follicles.

- Majocchi's granuloma

Generalised dermatophytosis

- Dermatophytic disease

- The above classification reflects the host parasitic relationship in dermatophytosis and gives a clue to logical therapy.

\section{CLINICAL FEATURES}

The clinical features of dermatophyte infections result from a combination of keratin distraction and inflammatory host response. Atypical lesion of dermatophytosis is an annular scaling patch with a raised margin showing a variable degree of inflammation, the centre usually being less inflamed than the edge. The wide variation in clinical presentation depends upon the species and probably the strain of fungus concerned, size inoculum, site of body infection and immune status of the host. ${ }^{[17,21]}$

\section{Tinea corporis}

Synonyms: Tinea circinata, Tinea glabrosa, Ringworm of the body

Definition: Ringworm of the body, usually involving the trunk, shoulders, or limbs and occasionally the face (excluding the beard area), may be caused by the any dermatophyte. ${ }^{[17]}$

The three most common causative organisms are Trichophyton rubrum, Microsporum canis and trichophyton mentagrophytes, variation may occur based on the existence of endemic species in specific geographic areas. India as reported by most workers and Trichophyton rubrum is the largest single etiological agent as reported from many parts of the country. ${ }^{[14]}$ The infection is more common in adult males. Children appear to have an increased incidence of Tinea corporis caused by zoophilic organism. ${ }^{[21]}$

Clinical Features: The clinical manifestation of Tinea corporis are varied and often depend on the species of the infective organism. The characteristic lesion is a circular, usually sharply marginated pink to slightly erythematous patch with a raised edge. As the lesion progresses, central clearing occurs and lesions takes an annular shape. The advanced border is more or less scaling, but in inflammatory forms, in general caused by Zoophilic or Geophilic dermatophytes, crusts, vesicles, papules or even pustules can develop. 
The degree of inflammation varies depending on the species of the fungus, the host's immune response and the extent of follicular invasion. Pustules and vesicles are seen in inflammatory lesions, while in less inflammatory lesions, scaling is the most prominent finding. ${ }^{[17,18,19,22]}$ Infections with Trichophyton rubrum can take this aspect with very chronic, non-inflammatory, extensive lesions. ${ }^{[17]}$ The central skin may show post inflammatory pigmentation, a change of texture or residual erythematous dermal nodules.

\section{Various clinical expressions of Tinea corporis infections}

Tinea Imbricata (Tokelau) is a specialized manifestation of Tinea corporis. The causative organism is the anthropophilic Trichophyton concentricum. The skin lesions are characteristically concentric and lamellar plaques of scale, scattered throughout the body, resembling erythema. The disease gets its name imbricata from this clinical pattern. ${ }^{[17,18,21]}$

Tinea corporis Gladiatorum is a clinical variant of the Tinea corporis which occurs among the competitive wrestlers, most out breaks are caused by Trichophyton tonsurans via person to person contact. Characteristic lesions are central clearing with a rough scaly circular border. Lesions may vary from small patches to large patches. ${ }^{[23,24,25]}$

Tinea incognito: It refers to a dermatophytic infection that has been modified clinically by the use of the oral or topical corticosteroids. Patient are generally immunosuppressed, in most cases, the annular scaling and the circumscribed border are absent, leading to a diffuse erythema with follicular papules. ${ }^{[17,21,22]}$

\section{VARIANTS OF TINEA CORPORIS BASED ON MORPHOLOGY:}

Annular Lesion: "Classic Ringworm" the lesion being usually as an erythematus papule, then enlarges to form an annular lesion with a relatively normal centre and a sharply defined active elevated red and infiltrated border.

Plaque Type: Chronic extension result in the formation of large scaling dull red plaque with sharply defined borders. Trichophyton rubrum is the chief offender.

Herpetiform Type: This is an inflammatory vesicular type of ringworm, usually due to a zoophilic species. The primary lesion is a mound of fused vesicles, which raptures living a red, eroded base on which a crust may form in the periphery.

Keroin Type: Keroin of the gabbros skin is usually due to a zoophilic organism Trichophyton verrucosum or Tricophyton montagrophytes. The lesion is intensely inflamed, consisting of an elevated, sharply circumscribed, buggy swelling with a bright red, exuding granulating surface, follicular pustule are a prominent feature. The suppuration is an evidence of reaction to the fungus although secondary bacterial infection may occurs later as the lesion ulcerates. ${ }^{[23]}$

Majocchi's Granuloma: This variant is essentially a granulomatous folliculitis and a perifolliculitis, Patient usually present with one or more tender buggy papules or plaques on the legs pus may be seen draining from the hair follicle. ${ }^{[17,19,22]}$

Mycetoma: This is a recalcitrant subcutaneous infection in which draining sinuses may be seen. Causative agents are Micosporum audouinii, Trichophyton verrucosum, Tricophyton mentagrophytes, Trichophyton violaceum, Trichophyton, tonsurans, Micosporum cannis, Trichophyton rubrum. ${ }^{[26]}$

\section{Tinea cruris}

Synonyms: Ringworm of the groin, dhobi itch, jock itch.

Definition: Tinea cruris is an acute to chronic inflammatory reaction of the skin of the inguinal region caused by dermatophytes. It includes infection of the genitalia, pubic area, perineal skin areas, and occasionally the upper thighs.

The predominant cause is anthropophilic species, Trichophyton 
rubrum most commonly, but Trichophyton interdigitale is not rare.

Adult are much more infected than children and the disease is three time more common in man than female, however, it has become more common in post pubertal females who are overweight or who often were tight jeans or pantyhose. ${ }^{[6,17,18,21,24]}$

Clinical Features: The lesions can begin unilateral, but very soon both groins are affected. Patches with erythema with central clearing are centred on the inguinal creases and take a semicircular aspect. Extension occurs both distally on the medial part of the thighs, and proximally to the lower abdomen and pubic area, the perineum and buttocks. The peripheral activity is characterised by fine scaling and the presence of some papules, vesicles and pustules. The border is sharply demarcated. In the more acute forms, the lesions may be moist and exudative or can have an eczematous aspect. In the chronic form, the lesions are dry with an annular or aciform aspect; some papules or small follicular nodules may be present and scaling is barely perceptible. $^{[6,17,21]}$

\section{Tinea Faciei}

Synonyms: Ringworm of the face, Tinea faciale, "Tinea corporis of the glabrous skin of the face"

Definition: It is the infection of the glabrous skin of the face with a dermatophyte fungus.

The causative agent varies according to geographic regions, but in general the source of the infection is a zoophilic reservoir in animals and pets or extension from an infection elsewhere on the body. The more common dermatophytes are Trichophyton rubrum and Trichophyton mentagrophytes.

Clinical Features: Tinea faciei often presents as erythematous, scaling, pruritic lesions on the face, some patient have the classical annular pattern of the Tinea corporis with discrete, raised, scaling borders, while other have indistinct flat erythematous patches. Itching and burning may become worse after exposure to sunlight. ${ }^{[17,18,21,22]}$

\section{Tinea Capitis}

Synonyms: Ringworm of the scalp, Tinea tonsuranse

Tinea capitis is a dermatophytosis of the scalp and associated hair and hair follicle. The infection can be located on the scalp, eyebrows, or eyelashes. The causative agents are fungi of the genera Trichophyton and Micosporum. Micosporum cannis is the dominant organism found worldwide. It is commonly more affected in children; boys are more affected than girls. The disease may develop as a consequence of shared headgear e.g. comb or hairbrush. ${ }^{[21,22,23]}$

Clinical Features: Clinical manifestations are varied and depend on the spaces of dermatophyte involved and the degree of host response. They infection may range from mild, almost subclinical, with single erythema and a few patchy areas of scaling with dull gray hair stump to highly inflammatory reaction with folliculitis, kerion formation, and extensive areas of scarring and alopecia, some time accompanied by fever, malaise, and regional lymphadenopathy.

There are four clinical patterns of

\section{Tinea capitis}

Seborrheic Pattern: it has the dandruff like scaling of the scalp often accompanied by itching and hair loss. Infection closely resembles seborrheic dermatitis. ${ }^{61,64}$

"Black dot" pattern: The endothrix infection causes the hair shaft to weaken and break off at the level of the scalp, living on the black dot on scalp; black dot is seen within the areas of alopecia. This pattern is primarily associated with Trichophyton tonsurans infection.

Kerion Pattern: A kerion is the painful inflammatory mass, discharging pus, in which the hairs that remain are loose. Thick crusting with matting of adjacent hair is common. A large confluent lesion may develop that involves most of the scalp. Patients usually have posterior cervical lymphadenopathy. Secondary bacterial 
infection may play a part. In most of the cases this violent reaction results from zoophilic Trichophyton verrucosum or Trichophyton mentagrophytes var.

Favus Pattern: it is now formations of the scapulae are yellow cup shaped crusts around the hair shafts. Adjacent crusts enlarge to become confluent and form a yellow crusting mass. ${ }^{[17,18,19,21,22,23]}$

Despite the clinical pattern there are three microscopic patterns of the Tinea capitis

Endothrix: Infection is produced by Trichophyton tonsurans and Trichophyton violaceum that invade the inside of the hair shaft and are composed of fungal arthroconidia and hyphae.

Ectothrix: this infection produced by fungi that primarily invade the outside of the hair shaft, and destroy the cuticle.

\section{Tinea Barbie}

Synonyms: Tinea sycosis, Barber's itch, ringworm of the beard

Definition: Ringworm of the beard and moustache area of the face with invasion of coarse hairs seen in the adult males. In most of the cases the zoophilic exothrix fungi Trichophyton verrucosum and Trichophyton mentagrophyte are responsible for the type of the infection. And Micosporum canis is less common cause. Usually the infection is contracted by exposure to animals and transmission from person to person is via contaminated barber's razors or clippers.

Clinical Feature: As with the Tinea corporis there are the variation of presentation ranging from the lesion with follicular pustules to non inflammatory scaly patches. ${ }^{[6,17,18,22]}$

Inflammatory or kerion like: most often caused by Trichophyton montagrophyte and Trichophyton verrucosum; this variety is analogous to kerion formation on the Tinea capitis. Nodular and buggy swelling associated with seropurulent discharge and crusting are present commonly on the chin, neck and maxillary and submaxillary area. The hair is loose and easily pluckable.
Scaring and permanent alopecia are the end results. ${ }^{[20]}$

Sycosiform type or Superficial: this type is manifest to diffuse erythema with perifollicular papule and pustule and resemble the bacterial folliculitis.

Atypical lesion: Atypical form like granuloma annulare like or abscess like tumours with Micosporum cannis and various granulomatous lesions with Epidermophyton floccosum may be recognised.

\section{Tinea Manuum}

Synonyms: ringworm of the hand

Definition: Tinea manuum is infection of skin and the interdigital skin of the hand. Infection of the dorsal surface is considered as ringworm of the glabrous skin. If both hand are affected the term Tinea manuum are used. The most common cause of the infection is Trichophyton rubrum, and most of the cases, there is pre-existing foot infection with or without nail involvement.

Clinical Features: The infection is usually unilateral, the right hand being more commonly affected than the left. Lesion on the dorsum appears similar to those of Tinea corporis. This person diffuses hyperkeratosis, with accentuation of fissuring in the palmar crease. ${ }^{[17,18,21,22]}$

There are two main cause of Tinea manuum Inflammatory: Inflammatory lesion can be found when other dermatophytes are involved. Vesicles usually multiloculated occur in clusters principally on the palms but rarely on the dorsal surface.

Non-inflammatory: This present as a mild asymptomatic scaling to diffuse scaling hyperkeratosis, with fissuring in palmar crease. ${ }^{[17,1822]}$

\section{Tinea Pedis}

Synonyms: Foot Ringworm, Athlete's foot Definition: It is the Dermatophyte infection of the plantar surface and interdigital space. The main organism involved is the anthropophilic species Trichophyton rubrum and Trichophyton mentagrophytes var interdigitale and Epidermophyton 
floccosum. Trichophyton rubrum is often associated with chronic non inflammatory, erythematosquamous reaction, infection due to Trichophyton mentagrophytes var interdigitale often lead to vesicular or bullous inflammatory lesions. ${ }^{[6,17,22]}$ It is more common in adolescents but rare in prepubertal children. The most important predisposing factor to acquiring the Tinea pedis appear to exposure to a moist environment and maceration of the skin, and particularly common in institution or places where common bathing facilities are used, the infection may be less prevalent in societies that do not commonly where shoes. ${ }^{[17,18,24]}$

\section{Clinical Features:}

Most inflammatory reactions result in vesicular-bulbous or even pustular lesions. Lack of inflammation is characterised by pink to red discoloration and diffuse scaling. The clinical manifestation is also influenced by external conditions such as hot, moist climates, hyperhidrosis and wearing shoes. ${ }^{[17]}$

The clinical presentation of the infection is altered in the patient with $\mathrm{T}$ lymphocyte abnormalities, including those with quire immunodeficiency syndrome, in whom there is often extensive spread of the lesion onto the dorsal surface of the foot. ${ }^{[21]}$

There are four clinical morphologies of Tinea pedis

Interdigital Tinea Pedis: It is the most common clinical form commonly referred to as "athlete's foot", is a dermatophytic infection involving the web spaces of the feet. The clinical presentation may be characterised by dryness, scaling and fissuring or by white, moist maceration. Irritation and itching are often present. ${ }^{[6,17 \text {, }}$ 18]

Chronic, Erythematosquamous Tinea Pedis: In the mildest form, a few small scaling collateral can be found, in more severe forms, diffuse, dry silvery white scaly lesions, covering a mild pink to red inflamed skin, are present. In the most severe cases, the soles, heels and sides of the feet are affected. ${ }^{[6,17,18]}$
Vesicular Type of Tinea Pedis: It is a dyshidrotic reaction of the sole often appears in patients suffering from months or years with interdigital Tinea pedis. This vesicular reaction may be limited. Vesicles may rupture, living a fine collaret, scaling and then heal spontaneously. When new vesicle appears, the clinical aspect of the healing and vesicular lesions can talk an eczematous appearance. ${ }^{[6,17,18]}$

Ulcerative Type: it is the secondary maceration and overgrowth of the bacterial flora commonly complicates the eruption leading to an inflammatory mixed process. Generally, ulceration in the web space with secondary bacterial infection occurs in the immuno-compromised patients with interdigital Tinea pedis. ${ }^{[6,17]}$

\section{Tinea Unguium}

Synonyms: Ringworm of the nails (Onychomycosis)

Definition: Invasion of the nail plate by dermatophyte is referred to as Tinea unguium; infection of the nail by non dermatophytic infection is called as onychomycosis. The latter word is often used as a general term for a nail infection. Toenails and finger nails can be infected; but toenail are more affected. All ten nails can be infected. But it is not rare to see that some nails are spared. Tinea unguium is a common cause of nail dystrophy however; it should be differentiated from other acquired and congenital conditions.

Trichophyton rubrum accounts for $70 \%$ of all cases and Trichophyton mentegrophytes var interdigitale for $20 \%$. In rare occasions, other species are involved.

Clinical Features: Onychomycosis can occur at any age, although it is more common with increasing age, male and female are equally affected.

Tinea of toenails is more common than the Tinea of fingernails, probably owing to the warm moist environment of the shoe, as well as trauma. Immunosuppression, hyperhidrosis, diabetes mellitus and decries vascular supply with 
aging are other factors. Tinea unguium is uncommon before puberty or in normally menstruating women. ${ }^{[22]}$

\section{Histopathology}

Dermatophyte infection tends to be restricted to the Horney epidermal layers of the skin and to the nail and hair. In Tinea capitis, infection begins with hyphal penetration of the stratum corneum of the scalp. Several weeks later, the fungus colonised the base of the hair follicles and penetrated the medulla of the hair shaft. The newly keratinised material of the growing hair, extending through the aperture of the follicle, carriers either within it or on and just beneath its cuticle's surface (ectothrix) hyphae that round up and become converted into arthroconidia.

In Tinea favosa of the scalp, filaments, often empty or vacuolated, are seen within infected hairs, while the scalp bear conspicuous cup-shaped areas of densely interwoven mycelium, scale and debris referred to as scutula. Inflammation with round cell infiltrate is seen in the adjacent dermis. Infected hair follicle tends to atrophy. In affected skin, peripherally raised and centrally depressed areas of the scutula tend to form.

Development of a hypersensitive, kerion reaction on the scalp or similar Tinea profounda lesion elsewhere is accompanied by extensive infiltration of lymphocytes, plasma cells, neutrophils and eosinophils into the dermis. Other features may include penetration of hyphae into the dermis and perivascular and perifollicular inflammation.

In the Tinea corporis, cylindrical fungal filament are seen in affected areas and rounded arthroconidia may develop. Affected skin may develop vesicles and papules accompanied by dermal infiltrates in infection caused by the zoophilic dermatophytes, while dry, scaly lesion and start from a proximal subungual onychomycosis. ${ }^{[17]}$

Marked by hyperkeratosis are characteristic of chronic infections caused by the anthropophilic dermatophytes. In an acute form Tinea pedis may reveal intercellular edema and leukocyte infiltrate in the epidermis, or in a chronic state, it may show hyperkeratosis and acanthosis.

Folliculitis caused by the Trichophyton rubrum is characterized by the presence of the fungal element in the follicles and a sign of a foreign body reaction, with foreign body giant cell in dermal infiltrates granuloma formation may occurs after minor trauma due to shavings of the legs.

The common digital subungual is characterised by the presence of the cylindrical fungal filaments and rounded arthroconidia penetrating between the lamellae of the lower nail plate proximal with subungual onychomycosis occurs almost exclusively in immunodeficient patient. The white patches initially limited to the lunula may eventually involve the entire nail. Superficial white onychomycosis by contrast is restricted to the surface of the nail and is characterised by the presence of irregular hyphae, often with flattened, spreading, frondose branching.

\section{LABORATORY DIAGNOSIS}

Because of the difficulty to differentiate clinically dermatophytosis from other non-mycotic dermatosis, particularly in the dystrophic nails, it is important to establish biologically an accurate diagnosis. A definitive diagnosis of the dermatophyte infection needs to be done before the initiation of the antifungal therapy. Some authors recommended the clinging the lesion area with alcohol before sampling to remove contaminates such as bacteria. ${ }^{[28]}$

The spacemen is placed on glass slide to which one or two drops of a 10-20\% $\mathrm{KOH}$ solution is added and a cover slip applied and allows remaining at room temperature for $15-20 \mathrm{~min}$. This allows $\mathrm{KOH}$ to dissolve the keratinous material, leaving the hyphae and spore more easily visible. The dermatophytes appear as hyaline, septate, branched and unbranched 
hyphae. Nail hyphae are slow to clear; the full thickness nail clipping may be placed in a weak $5 \% \mathrm{KOH}$ solution in a watch glass for 24 hours to obtain the adequate clearing. During examination of sample, the condenser of the microscope should be in the down position. The presence of the septate hyphae conform the diagnosis of the Tinea. The examiner makes sure that hyphae are being seen rather than the edge of an epithelial cell. It is helpful to visualize the hyphae crossing the path of more than one cell. ${ }^{[29]}$

\section{Wood light Examination}

Visualisation of the fungal elements at direct examination is some time difficult staining can increase sensitivity of direct examination by facilitating the visualization of the fungal structure. Various stains which can be associated with clearing agent may be used. For example, chlorazol black Stain only the fungal structure and exclude many artefacts. Likewise, blue-black ink permanent which stain the fungal elements in deep blue, or cotton blue $\mathrm{C} 4 \mathrm{~B}$ may be used. Congo red also can be used for direct examination this stain bind the polysaccharides of the fungal cell wall, particularly b-D glucans, and therefore facilitates the detection of fungal elements. Calcofluor-stained specimens appear blue when using a Florence microscope equipped with a 330-380 $\mathrm{nm}$ excitation filter and an emission filter of $\geq 420 \mathrm{~nm}$. However, green fluorescence may be observed with fluorescence filter system. ${ }^{[28]}$

\section{MANAGEMENT}

\section{Treatment Depending on Morphology of the Disease}

The choice of treatment and its duration depends on the causative Organism, the site of infection and the extent of the disease, as well as on the other factors for each individual patient, such as concurrent disease and medication. ${ }^{\text {[21] }}$

The main topical agent used for Dermatophytosis is the keratolytics and compounds with specific antifungal activity.
The azoles, which include miconazole, clotrimazole, econazole, tioconazole, ketoconazole, oxiconazole, bifonazole, isoconazole, and fenticonazole. Other potent antifungal uses are Ciclopiroxolamine, Terbinafine, and butinafine. ${ }^{[21]}$

Until recently, Griseofulvin has been the mainstay of oral therapy. Ketoconazole is also effective against the dermatophyte fungi, but its use has been limited by the potential, but rare risk of Hepatotoxicity. Evolving therapy include itraconazole, fluconazole, and oral terbinafine. ${ }^{[22]}$

\section{Tinea Capitis and Tinea Barbae}

The treatment consists of the systemic antimycotic therapy. Griseofulvin is the drug of choice and is generally dose 15 to $25 \mathrm{mg} / \mathrm{kg} /$ day and will ultra micronized griseofulvin 10 to $15 \mathrm{mg} / \mathrm{kg} / \mathrm{day}$. It is administered after fatty meal or milk. It is given preferably at night and it has been known to cause the photosensitivity. [30] Fatty meals enhance the absorption. Treatment should generally be continued until be patient is clinically and mycologicaly cured, which can take 6-8 weeks or even longer in some patients. Other effective agents are fluconazole (3to 6 $\mathrm{mg} / \mathrm{kg} /$ day for 6 weeks), itraconazole (3-5 $\mathrm{mg} / \mathrm{kg} /$ day $)$, and terbinafine $(250 \mathrm{mg} /$ day fore body weight more than $40 \mathrm{~kg}, 125$ $\mathrm{mg} /$ day for body weight between the 20 and 40 , and $62.5 \mathrm{mg} /$ day for weight less than 20 $\mathrm{kg}$ ). Plus therapy for itraconazole has been reported by Gupta et al. ${ }^{[31]}$ Pulse therapy with terbinafine has also been reported. In one study, each pulse of active therapy lasted for one week, with two week of between first two pulses and three week off between the second and third pulses. While in other study, there was a three week off period between active pulse therapies of one week's duration. [32] Topical therapies should be used parallel to systemic therapy.

Systemic or topical corticosteroids may also be of benefit to patients with inflammatory Tinea capitis to avoid scarring and permanent hair loss. 


\section{Tinea Corporis Tinea Cruris, Tinea Faciei and Tinea Pedis}

Topical therapy is generally given in these types. Generally the topical agents should be applied twice daily for 2-4 weeks. The newer allylamine terbinafine however has been reported effective in 1 week of therapy, possibly attributed to its fungicidal property. Shorter courses of therapy lead to increased compliance. Premature discontinuation of therapy leads to treatment failure. In patients with extensive disease, those with inflammatory infections, and in those who are immunocompromised, 1-2 weeks course of systemic ketoconazole, griseofulvin, or one of the never triazoles; fluconazole or itraconazole or systemic terbinafine might also be helpful as adjunctive therapy. Occasionally, topical corticosteroids help relieve pruritus and inflammation.

Tinea Unguium: For Tinea unguium, the most resistant to dermatophytosis, topical therapy is seldom efficacious and spontaneous resolution is rare. One long standing strategy is avulsion or chemical ablation of the nail, followed by treatment of the nail bed with fungistatic agents. Chemical ablation is often accomplished with urea paste under occlusion. ${ }^{[27]}$

Topical application of tioconazole or amorolfine should be continued for at least 6 month for finger nail and 12 month for toe nail. Oral terbinafine is the treatment of choice for proven Tinea unguium. Terbinafine is administered $250 \mathrm{mg} /$ day for 6 week in the finger nail and 12 week in toe infections. Similarly, the recommended itraconazole regimen for toe nail with or without finger nail involvement is 200 $\mathrm{mg} /$ day for 12 week. ${ }^{[24,27]}$

A pulse regimen consisting of 1 week treatment of 1 week treatment period with $200 \mathrm{mg}$ twice daily separated by a 3week drug free period per month for 2 month, both in finger nail and toenails, or 3 or 4 consecutive months in toe nails. ${ }^{[24,27]}$

\section{PREVENTION AND CONTROL}

Prevention and control of dermatophytic infections must take into consideration the area invaded, the etiologic agent, and the source of infection. Routine inspection of scalps of young children should be performed at the beginning of the school term. All outbreaks in schools or institutions should be reported to the proper authorities. Good hygiene should be impressed upon those infected, and they must be instructed not to shar headgear, combs, and brushes. Barber shop instruments (combs, scissors, and brushes) must be disinfected after use. All those infected must be treated promptly to prevent further spread of the infection.

Since Tinea corporis and Tinea cruris caused by Anthropophilic fungi can be transmitted by infected clothing, towels, and bedding, these items should be disinfected after use by infected individuals should not permit others to share them. Individuals with Tinea corporis should not engage in contact sports such as wrestling.

It is important to locate the animal reservoir in infection caused by the zoophilic dermatophytes. Good hygiene and sanitation and fungicidal sprays and washes have been effective in controlling these infections. When economically feasible, systemic griseofulvin could be used to treat infected cattle.

Prevention of the Tinea pedis may be enhanced by using good food hygiene (includes regular wishing of the feet, through drying, and application of foot powder), avoiding excessive moisture and occlusion by wearing sandals or other well ventilated shoes, avoiding trauma to the feet, Since Tinea pedis is considered contagious, i.e., transfer by infected shed skin scales, control may be accomplished by educating infected individuals not to expose other by walking bare foot near swimming pools, locker rooms, and public showers and by not sharing footgear.

According to some investigators, an individual carries pathogenic fungus in quiescent foci on their nails, feet, and groin 
infection exacerbates when trigger factors lower resistance. Measure for prevention should be based on maintenance of local resistance to infection by individual care and hygiene of the feet and groin.

\section{Acknowledgement: None}

\section{Conflict of Interest: None}

\section{Source of Funding: None}

\section{REFERENCES}

1. Chin, Y.W., Balunas, M.J., Chai, H.B., Kinghorn, A.D., (2006). Drug Discovery from Natural Sources. AAPS Journal 8: E pp. 239-253.

2. Verma S, Heffernan MP. Superficial fungal infection: dermatophytosis, tinea nigra, piedra. In: Fitzpatrick TB, Eisen AZ, Wolff $\mathrm{K}$, Freedberg IM, Austen KF, editors. Dermatology in general medicine. 7th ed. New York: McGraw-Hill; 2008. P.1807-21.

3. Lacaz CS, Porto E, Martins JEC, HeinsVaccari EM, Melo NT. Tratado de Micologia Médica Lacaz. 9 ed. São Paulo: Sarvier; 2002. p.252-340.

4. Hay RJ, Ashbee HR. Superficial mycoses. In: Burns T, Breathnach S, Cox N, Griffiths C, editors. Rook's Textbook of Dermatology. 8 th. Singapore: Wiley Blackwell; 2010. p. 36.5-36.68.

5. Peres, N.T., Maranhao, F.C., Rossi, A., Martinez-Rossi NM., (2010). Dermatophytes: Host Pathogen Interaction and Antifungal Resistance. An Bras Dermatol. 85: pp. 657-667.

6. Weitzman, I., Sumberbell, R.C., (1955). The Dermatophytes Clinical Microbiological Reviews, 8, pp. 240-259.

7. Johnson Liz., (2003). Dermatophytes, the skin Eaters, Mycologist, pp. 147-49.

8. Simpanya M.F., (2000). Dermatophytes, there Taxonomy, Ecology and pathogenicity Spain, Revista Iberoamericana de Micologia Apdo.

9. Havlikova, B., Czaika, V.A., Friedrich, M., (2008). Epidemiological Trends in Skin Mycoses Worldwide. Mycosis, 51. 2-15.

10. Bassiri-Jahromi, S., Khaksari, A.A., (2009). Epidemiological Survey of Dermatophytosis in Tehran, 2000 to 2005 Indian J Dermatol, Venrol, Leprol, 75: pp.142-7.
11. Jerajani, H.R., Amladi, S.T., Bongale, R., Adepu, V., (2000). The Evaluation of Clinical Efficacy and Safety of Once Daily Topical Administration of 1\% Oxiconazole cream and Lotion of the Dermatophytosis, an Open Label Non Comparative Multicentre Study. Indian J. Dermatol, Venrol, Leprol, 66: pp.188-92.

12. Jochen Brasch., Uta-Christina Hipler., (2008). Clinical Aspect of the Dermatophytes Infections, Human and Animal Relationships. The Mycota 2ed ed. VI Berlin Heidelberg: Springer-Verlag.

13. Raza Aly., (1994). Ecology and Epidemiology of Dermatophyte infections. Journal of the American Academy of Dermatology, 31: S21-S25.

14. Garage, J., Tilak R., Garg A., Parkash P., Gulati A.K., Nath G., (2009). Rapid Detection of Dermatophytes from Skin and Hair BMC Research Notes, 2: pp.60.

15. Thappa, D.M., Textbook of Dermatology, Venereology and Leprology. $3^{\text {rd }}$ ed. Gurgaon, Elisevier Publications, pp. 76.

16. Greer, D.L., (1994). An Overview of Common Dermatophytes. J Am Acad Dermatol, 31: pp. 112.

17. Degreef, H., (2008). Clinical forms of Dermatophytosis, Mycopathologia, pp.166, 257-265.

18. Donald, A., (1999). Jonathan Clinical Infection Diseases, Barcelona Harcourt Publisher Ltd, pp. 2: 6.1-6.6.

19. Fitzpatrick James E., Morelli Joseph G., Dermatology Secrets in Colour. $3^{\text {rd }}$ ed. New Delhi, Elsevier, pp. 253-60.

20. Behl P.N., Aggarwal A., Srivastava G., (2004). Practice of Dermatology. $9^{\text {th }}$ ed. New Delhi, CBS Publisher, pp. 164-71.

21. Mandell Gerald, L., Bennet john, E., Raphael, D., (2005). Principles and Practice of Infectious diseases, Pennsylvania, elsevier Churchill Livingstone, Vol, 2. $6^{\text {th }}$ ed, pp 3051-3059.

22. Sams, W., Mitchell, Lynch Peter, J., (1996). Principle and Practice of Dermatology. $2^{\text {nd }}$ ed. Singapore, Churchill Livingston, pp. 149-57.

23. McKee Phillip, H., Calonje, E., Granter Scott R., (2005). Pathology of the Skin with Clinical Correlation. Vol-I, $3^{\text {rd }}$ ed. London, Elsevier, pp. 939-43.

24. Andrews, M.D., Burns, M., (2008). Common Tinea Infections in Children, American Family Physician, pp. 1415-20. 
25. Bassiri-Jahromi S, Khaksar, A.A., (2008). Outbreak of Tinea Gladiatorum in Wrestlers in Tehran, Indian Journal of Dermatology, 53 (3): pp.132-6

26. Shah Siddharth, N., (2003). API Text Book of Medicine, Mumbai, The Association of Physician of India, 7th ed. pp.1304-1305.

27. Haber, R.M., (2007). Dermatological Fungal Infections. The Canadian Journal of Diagnosis, pp. 64-68.

28. Robert, R., Pihet, M., (2008). Conventional Methods for the Diagnosis of the Dermatophytosis, Mycopathologia, pp. 166, 295-306.

29. Anderw, W., Barian, B., (2002). Topical Treatment of Common Superficial Tinea Infections, American Family Physician, pp. 2095-2102.

30. Chan, K.T., David, W.S., Cheng, C., Guan, P.S., Koh, M.H., Wong, S.W., (2013). Anti-
Allergic Actions of Rottlerin from Mallotus philippinensis in Experimental Mast CellMediated Anaphylactic Models, Phytomedicine, Vol. 20, no. 10, pp. 853860.

31. Maeda, (2009). Method for Producing Mallotus philippinensis Dye Composition and the Composition, US7556829 B2.

32. Wiart, C., (2006). Medicinal Plants of the Asia-Pacific, Drugs for the Future World Scientific, Singapore.

How to cite this article: Ahmad S, Ahmad G, Mohsin M et.al. Superficial dermatophytic infection prevention and its management: a review. International Journal of Research and Review. 2021; 8(8): 427-439. DOI: https://doi. org/10.52403/ijrr.20210859 